\title{
Interpretation of the Ni K-edge EXAFS in nanocrystalline nickel oxide using molecular dynamics simulations
}

\author{
A. Anspoks, A. Kuzmin* \\ Institute of Solid State Physics, University of Latvia, Kengaraga street 8, LV-1063 Riga, Latvia
}

\begin{abstract}
Analysis of atomic structure at the nanoscale is a challenging task, complicated by relaxation phenomena and thermal disorder. In this work, the X-ray absorption spectroscopy at the Ni K-edge was used to address this problem in nanocrystalline NiO (nano$\mathrm{NiO})$. The expansion of the average lattice but contraction of the $\mathrm{Ni}-\mathrm{O}$ bonds in the first coordination shell were determined in nano-NiO at $300 \mathrm{~K}$ in comparison with bulk material. Accurate EXAFS analysis, based on a combination of classical molecular dynamics and ab initio multiple-scattering EXAFS theory, allowed us to interpret full EXAFS spectrum. In particular, the effect of magnetostriction effect was elucidated in bulk NiO, and the effect of the thermal disorder in outer coordination shells was studied in both bulk and nano-NiO.
\end{abstract}

Keywords: NiO, Ni K-edge, EXAFS, Molecular dynamics, Nanoparticles PACS: 61.05.cj, 78.70.Dm, 61.46.Hk, 61.43.Bn

\section{Introduction}

Determination of atomic structure at the nanoscale is far from trivial task, complicated additionally by atomic structure relaxation. The problem can be addressed within a complex modelling approach combining the experimental results and the theory in some effective way [1]. Among different experimental techniques applicable to nanostructured materials, only scattering/diffraction methods and $\mathrm{x}$-ray absorption spectroscopy (EXAFS) provide with the direct structural information. The former methods, gaining popularity for nanostructure studies under the name of the total scattering/PDF analysis [2], contain information on the atomic pair distribution functions (PDFs). At the same time, the EXAFS spectra include also contributions from many-atom distribution functions, which, however, are very difficult to access [3]. Therefore, it becomes a challenging task to extract the three-dimensional nanoparticle structure from the total EXAFS signal.

Chemical and physical properties of nanoparticles are directly connected to their shape and size [4]. The reduction in size causes usually the atomic structure relaxation, leading to compression or expansion of nanoparticles volume. While the volume compression is common for metal nanoparticles, its expansion is observed in most nano-metal-oxides [5, 6, 7, 8, 9]. In particular, the lattice expansion has been found recently by $\mathrm{x}$-ray diffraction $[10,11]$ in nanocrystalline $\mathrm{NiO}$, having a size below about $30 \mathrm{~nm}$.

In this work we present the application of recently developed approach [12] to the interpretation of the Ni K-edge EXAFS

\footnotetext{
${ }^{*}$ Corresponding author.

Email address: a.kuzmin@cfi.lu.lv (A. Kuzmin)

URL: www.cfi.lv (A. Kuzmin)
}

in nanocrystalline $\mathrm{NiO}$, having a size of about $13 \mathrm{~nm}$. Our method is based on a combination of classical molecular dynamics (MD) and ab initio multiple-scattering EXAFS theory. The use of MD allows to constrain a structural solution by introducing just a few force field model parameters and to gain an access to both structure and dynamics of the nanocrystal. A set of atomic configurations, generated from the MD run at desired experimental conditions, is further used to obtain configuration averaged EXAFS signal, whose agreement with the experimental data is used as a proof of the MD model reliability.

\section{Experimental and data analysis}

Black $\mathrm{NiO}$ nanoparticles (nano-NiO) were produced using a reaction of aqueous solutions of $\mathrm{Ni}\left(\mathrm{NO}_{3}\right)_{2} \cdot 6 \mathrm{H}_{2} \mathrm{O}$ salt and $\mathrm{NaOH}$ with subsequent annealing in air at $300^{\circ} \mathrm{C}$. The average size of nanoparticles was $13 \mathrm{~nm}$ according to the BET specific surface area measurements [18]. The nanocrystallites size of $6.5 \pm 1.5 \mathrm{~nm}$ was estimated from the line broadening of the $\mathrm{X}$ ray diffraction (111), (200), and (220) peaks, corrected for instrumental broadening, using the Scherrer method and assuming the cubic crystallites shape. The commercial green polycrystalline nickel oxide (c-NiO, Aldrich, 99\%) was used for comparison.

The Ni K-edge EXAFS signals were measured in transmission mode at the HASYLAB DESY C1 bending-magnet beamline at $300 \mathrm{~K}$. The storage ring DORIS III operated at $E=4.44 \mathrm{GeV}$ and $I_{\max }=140 \mathrm{~mA}$ in a five bunches mode with a lifetime of $4 \mathrm{~h}$. The higher-order harmonics were effectively eliminated by detuning of the monochromator $\mathrm{Si}(111)$ crystals to $60 \%$ of the rocking curve maximum, using the beamstabilization feedback control. The $\mathrm{x}$-ray beam intensity was 
Table 1: Structural parameters for the first and second coordination shells in c$\mathrm{NiO}$ and nano-NiO obtained from the best-fit of the Ni K-edge EXAFS signals.

\begin{tabular}{|c|c|c|}
\hline & 1 shell $\left(\mathrm{Ni}-\mathrm{O}_{1}\right)$ & 2 shell $\left(\mathrm{Ni}-\mathrm{Ni}_{2}\right)$ \\
\hline & \multicolumn{2}{|c|}{$\mathrm{c}-\mathrm{NiO}$} \\
\hline$N$ & $6 \pm 0.2$ & $12 \pm 0.4$ \\
\hline$R(\AA)$ & $2.070 \pm 0.003$ & $2.949 \pm 0.003$ \\
\hline \multirow[t]{2}{*}{$\sigma^{2}\left(\AA^{2}\right)$} & $0.0057 \pm 0.0005$ & $0.0067 \pm 0.0005$ \\
\hline & \multicolumn{2}{|c|}{ nano-NiO } \\
\hline$N$ & $5.8 \pm 0.2$ & $11.3 \pm 0.4$ \\
\hline$R(\AA)$ & $2.061 \pm 0.003$ & $2.961 \pm 0.003$ \\
\hline$\sigma^{2}\left(\AA^{2}\right)$ & $0.0077 \pm 0.0005$ & $0.0082 \pm 0.0005$ \\
\hline
\end{tabular}

measured by two ionization chambers filled with argon and krypton gases. The powder samples for x-ray absorption measurements were deposited on Millipore filters to give an absorption edge jump $\Delta \mu x \simeq 1$.

The x-ray absorption spectra were analysed using the "EDA" software package [13]. The x-ray absorption near-edge structure (XANES) and the extended x-ray absorption fine structure (EXAFS) were extracted following conventional procedure [14] (Figs. 1 and 2). The EXAFS signals were modelled using two methods: (i) conventional multi-component approach within the single-scattering approximation [14], and (ii) recently developed MD-EXAFS approach, based on the use of classical molecular dynamics (MD) and ab initio multiplescattering theory [12].

In the conventional analysis, the contribution to the total EXAFS signal from the first two coordination shells $\left(\mathrm{Ni}-\mathrm{O}_{1}\right.$ and $\mathrm{Ni}-\mathrm{Ni}_{2}$ ) was first isolated by the Fourier filtering procedure in the $R$-space range of 0.7-3.2 $\AA$. Next, the two-component Gaussian model within the single-scattering approximation was used to best-fit the obtained EXAFS signal in the $k$-space range of 2-15 $\AA^{-1}$. To perform EXAFS calculations, one requires the knowledge of the scattering amplitude and phase shift functions for the $\mathrm{Ni}-\mathrm{O}$ and $\mathrm{Ni}-\mathrm{Ni}$ atom pairs. Here they were calculated by the ab initio FEFF8 code [15]. The calculations were performed for the cluster of $8 \AA$ size centred at absorbing nickel atom and having the cubic $\mathrm{NiO}$ structure [16]. The inelastic losses were taken into account using the complex exchange-correlation Hedin-Lundqvist potential [3]. The results of the best-fits are shown in Fig. 3. The obtained structural parameters, such as the coordination numbers $(N)$, the interatomic distances $(R)$ and the mean-squared relative displacements (MSRDs), known also as EXAFS Debye-Waller factors, $\left(\sigma^{2}\right)$, are reported in Table 1 .

\section{MD-EXAFS simulations}

To overcome the limitations of the conventional EXAFS analysis, we employed recently developed method [12], based on the calculation of the configuration-averaged EXAFS signal using atomic configurations generated within classical molecular dynamics (MD) simulation. Note that the method enormously reduces the number of free model parameters to that
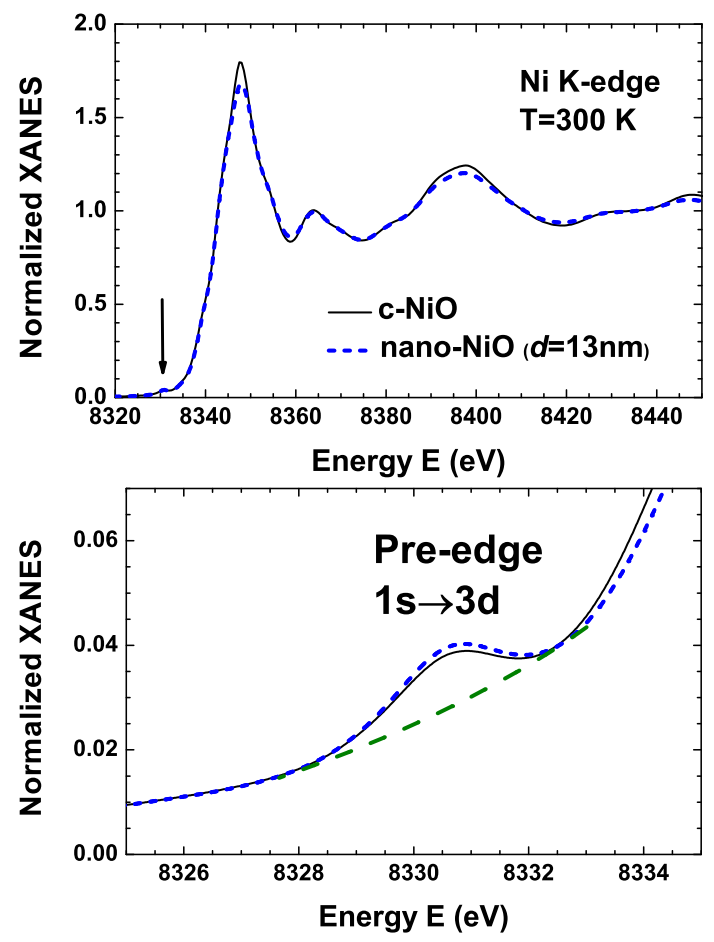

Figure 1: Upper panel: Experimental Ni K-edge XANES in c-NiO (solid lines) and nano-NiO (dashed lines) at $300 \mathrm{~K}$. The position of the pre-edge peak $A$ is indicated by arrow. Lower panel: enlarged region of the pre-edge peak $A$.
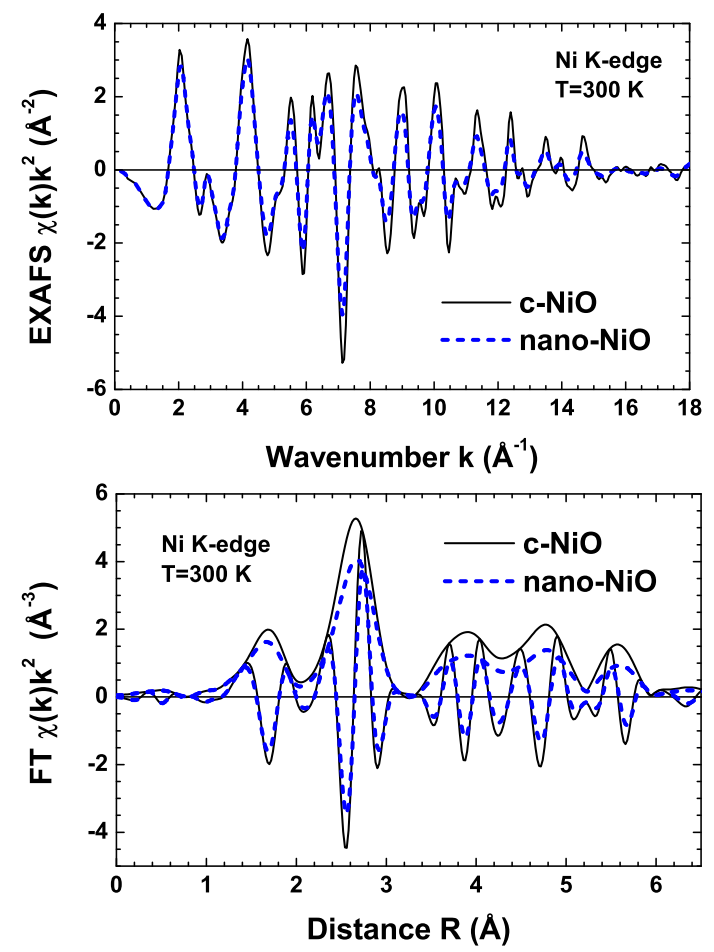

Figure 2: Experimental Ni K-edge EXAFS $\chi(k) k^{2}$ signals and their Fourier transforms for $\mathrm{c}-\mathrm{NiO}$ (solid lines) and nano-NiO (dashed lines) at $300 \mathrm{~K}$.

required by the force-field description $[12,17,19]$. 


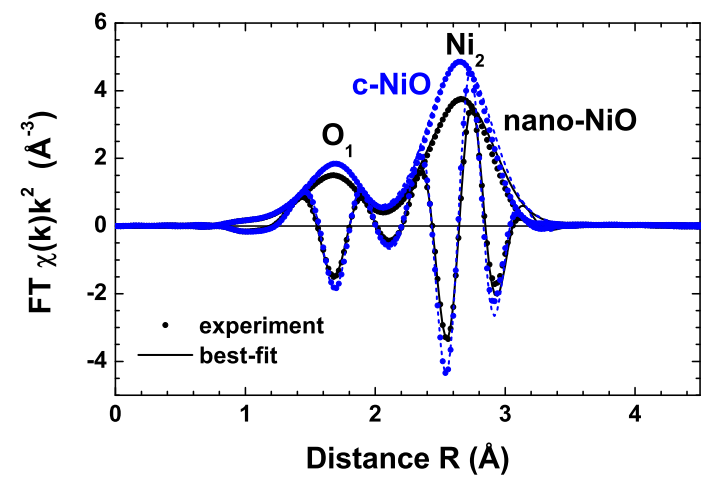

Figure 3: Fourier transforms of the experimental (open circles) and calculated (solid lines) Ni K-edge EXAFS $\chi(k) k^{2}$ signals for c-NiO and nano-NiO within the range of the first two $\left(\mathrm{O}_{1}, \mathrm{Ni}_{2}\right)$ coordination shells.

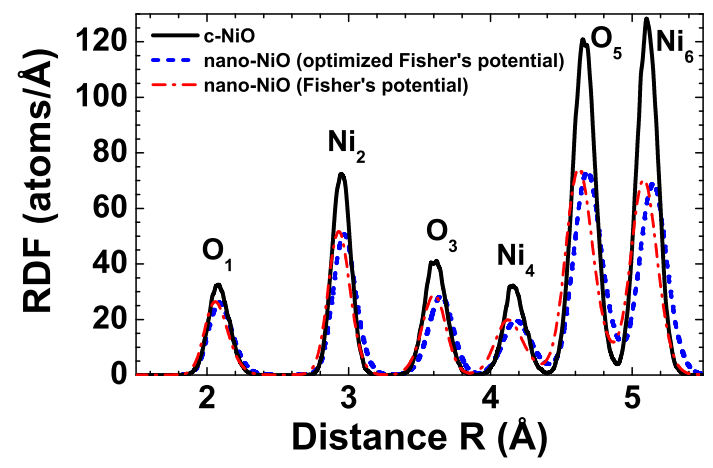

Figure 4: The total radial distribution functions (RDFs) $G(R)$ around absorbing nickel atoms for c-NiO (solid line) and nano-NiO (dashed lines - optimized Fisher's force field (FF); dash-dot line - Fisher's FF [23]) obtained from molecular dynamics simulations. Note the progressive coordination number reduction for nano-NiO. The first six coordination shells $\left(\mathrm{O}_{1}, \ldots, \mathrm{Ni}_{6}\right)$ are labeled.

First, we performed classical MD simulations in the canonical ensemble (NVT) by the GULP3.1 code [20], which is suitable for both crystalline and nanosized materials. Crystalline $\mathrm{c}-\mathrm{NiO}$ was modeled by the supercell $6 a_{0} \times 6 a_{0} \times 6 a_{0}$, where the lattice parameter $a_{0}=4.1773 \AA$ [16], with 3D periodic boundary conditions. The simulations for $\mathrm{c}-\mathrm{NiO}$ were also performed for rhombohedrally distorted ( $R \overline{3} m$ space group) lattice to check the effect of weak magnetostriction effect present in the antiferromagnetic phase below $523 \mathrm{~K}[21,22]$.

The nanosized $\mathrm{NiO}$ was simulated by a cluster having the cubic shape and the size of up to $6 a_{0} \times 6 a_{0} \times 6 a_{0}$. No boundary conditions were employed in this case. Our estimate for the cubic shape particles indicate that the EXAFS signal should feel the difference between crystal and nanoparticle when the particle size becomes smaller than about $400 \AA$. For larger particles, the contribution from atoms, located close to the surface and having reduced coordination, is too small to be detectable. Currently, the size of about $25 \AA$ is the largest accessible technically to our simulations.

The force-field (FF) potential model, based on the two-body central force interactions, was employed in the MD simulations. It consists of a sum of the Buckingham and Coulomb potentials
[20]

$$
U_{i j}\left(r_{i j}\right)=A_{i j} \exp \left(-r_{i j} / \rho_{i j}\right)-\frac{C_{i j}}{r_{i j}^{6}}+\frac{Z_{i} Z_{j} e^{2}}{r_{i j}} .
$$

The Buckingham potential parameters $(A, \rho$, and $C$ in Table 2$)$ were taken from available data [23, 24] for $\mathrm{c}-\mathrm{NiO}$. The formal ion charges $Z(Z=+2$ for $\mathrm{Ni}$ and $Z=-2$ for $\mathrm{O})$ were used in the Coulomb potential $[23,24]$. We found that the Fisher's FF model [23] gives slightly better agreement with the experimental Ni K-edge EXAFS signal for c-NiO than the Oliver's one [24], therefore only the former was used in the simulation of nano-NiO. However, the original value of the Buckingham potential parameter $\rho$ in the Fisher's FF model [23] was adjusted (Table 2) for nano-NiO to reflect the change in the frequency of the experimental EXAFS signal being related to the lattice expansion (Table 1).

The Newton's equations were integrated by the leapfrog Verlet method [20]. In each simulation, the structure was first equilibrated during $20 \mathrm{ps}$ at $300 \mathrm{~K}$, corresponding to the temperature of the EXAFS experiments, and a set of instantaneous atomic configurations was accumulated during the 20 ps production run with a time step of $0.5 \mathrm{fs}$. Thus obtained sets of instantaneous atomic configurations were used to calculate the total and pair radial distribution functions (RDFs) (Fig. 4), which were used to evaluate the values of MSRDs for the first and second shells.

Finally, the Ni K-edge EXAFS signals were calculated for each instantaneous atomic configuration using ab initio FEFF8 code [15]. The scattering potentials and partial phase shifts were evaluated only once for the average configuration, thus neglecting a variation of the scattering potentials due to thermal vibrations [12]. The multiple-scattering effects were taken into account up to the eights order with the half path length up to $6.5 \AA$. The inelastic losses were taken into account using the complex exchange-correlation Hedin-Lundqvist potential [3]. The cluster potential, used in the scattering amplitudes calculation, was of muffin-tin (MT) type, and the values of the MT-radii were $R_{\mathrm{MT}}(\mathrm{Ni})=1.319 \AA$ and $R_{\mathrm{MT}}(\mathrm{O})=1.021 \AA$. The configuration-averaged EXAFS signal was obtained by averaging over several thousands configurations (normally 4000), and its convergence was controlled. The calculations were performed using the cluster-type computer at ISSP [25].

\section{Results and discussion}

The normalized to the absorption edge jump Ni K-edge XANES signals for crystalline and nanocrystalline nickel oxide are compared in Fig. 1. They are composed of the low energy pre-edge peak A at $\sim 8335 \mathrm{eV}$, the main maximum at $\sim 8347 \mathrm{eV}$, and the fine structure above it. The two XANES signals are very close, except that the main maximum and the broad peak at $\sim 8400 \mathrm{eV}$ have slightly higher intensity in c-NiO. The preedge region of the XANES provides with the information on the local electronic structure at the absorbing $\mathrm{Ni}$ atoms in the presence of the core-hole. In particular, the Ni K-edge absorption is caused by the transition of a 1 s electron of nickel from its 
Table 2: Force field (FF) parameters of the Buckingham potentials for c-NiO and nano-NiO

\begin{tabular}{|c|c|c|c|}
\hline Pair of atoms & $A(\mathrm{eV})$ & $\rho(\AA)$ & $C\left(\mathrm{eV} \AA^{6}\right)$ \\
\hline $\mathrm{c}-\mathrm{NiO}$ & \multicolumn{3}{|c|}{ Oliver's FF [24] } \\
\hline $\mathrm{Ni}^{+2}-\mathrm{O}^{-2}$ & 775.0 & 0.3250 & 0.0 \\
\hline $\mathrm{O}^{-2}-\mathrm{O}^{-2}$ & 22764.3 & 0.1490 & 20.37 \\
\hline $\mathrm{c}-\mathrm{NiO}$ & \multicolumn{3}{|c|}{ Fisher's FF [23] } \\
\hline $\mathrm{Ni}^{+2}-\mathrm{O}^{-2}$ & 754.92 & 0.3277 & 0.0 \\
\hline $\mathrm{O}^{-2}-\mathrm{O}^{-2}$ & 22764.3 & 0.1490 & 27.89 \\
\hline nano-NiO & \multicolumn{3}{|c|}{ optimized Fisher's FF } \\
\hline $\mathrm{Ni}^{+2}-\mathrm{O}^{-2}$ & 754.92 & 0.3310 & 0.0 \\
\hline $\mathrm{O}^{-2}-\mathrm{O}^{-2}$ & 22764.3 & 0.1505 & 27.89 \\
\hline
\end{tabular}

core atomic state to a final unoccupied state following to dipole $(\Delta l= \pm 1)$ or quadrupole $(\Delta l=0, \pm 2)$ selection rules. Note that the final state is a relaxed excited state due to the presence of a 1s core hole screened by the other electrons of nickel.

The interpretation of the Ni K-edge XANES in $\mathrm{NiO}$ has been a topic of extensive studies in the past [26, 27, 28, 29, 30, 31, $32,33]$. The use of the multiple-scattering approach allowed understanding the origin of main peaks to be due to the scattering by atoms of the first ten coordination shells surrounding the absorber [26, 30]. The pre-edge peak A (Fig. 1) was assigned to the quadrupole $1 \mathrm{~s} \rightarrow 3 \mathrm{~d}$ transition from the analysis of resonant $\mathrm{X}$-ray magnetic scattering in [27]. Its quadrupole origin was also supported by several theoretical calculations [29, 31, 32].

In the recent work [33], the DFT $+U$ calculation scheme, i.e., the density-functional theory (DFT) with the Hubbard- $U$ correction obtained by linear response, within the spin-polarized generalized gradient approximation (GGA) was applied to the analysis of $\mathrm{Ni} \mathrm{K}$-edge in $\mathrm{NiO}$. It was found that the pre-edge peak A consists of two contributions: (i) a quadrupole part caused by the intrasite $1 \mathrm{~s}(\mathrm{Ni}) \rightarrow 3 \mathrm{~d}(\mathrm{Ni})$ excitations lowered by core-hole attraction, and (ii) a very small dipolar component due to the $3 \mathrm{~d}(\mathrm{Ni})-2 \mathrm{p}(\mathrm{O})$ hybridization between nearest $\mathrm{Ni}$ and $\mathrm{O}$ atoms [33]. At the same time, the next feature $\mathrm{B}$ of dipolar origin (Fig. 1) has nonlocal nature, caused by hybridization of on-site Ni empty $4 \mathrm{p}$ states with empty $3 \mathrm{~d}$ states of the $\mathrm{Ni}$ atoms located in the fourth coordination shell [33] and participating in the $180^{\circ} \mathrm{Ni}-\mathrm{O}-\mathrm{Ni}$ antiferromagnetic interactions. Due to the nonlocal origin of the feature $\mathrm{B}$, its position is insensitive to the core-hole attraction and, thus, the A-to-B peak separation can be used to estimate the value of the charge transfer gap in $\mathrm{NiO}$ [33]. The similarity of the XANES signals in bulk c-NiO and nano-NiO (Fig. 1) suggests the closeness of their electronic structures: in particular, one can conclude that no significant changes in the spin ordering should occur in $\mathrm{NiO}$ upon a decrease of the crystallites size down to $13 \mathrm{~nm}$.

The difference between the Ni K-edge EXAFS signals of c$\mathrm{NiO}$ and nano-NiO is much more pronounced than between their XANES signals: it appears mainly in the amplitude damping and a difference in the frequency of oscillations - both effects are better observed at high $k$-values (Fig. 2). The Fourier transforms (FTs) of the EXAFS signals demonstrate the progressive lowering of the peaks amplitude at larger $R$ values. Such behaviour is related to the nanosized effect in $\mathrm{NiO}[28,34]$, which includes both the reduction of coordination number due to an increase of the number of under-coordinated atoms at the nanocrystals surface and an increase of the disorder due to the nanoparticles structure relaxation.

First, the best-fit analysis of the EXAFS signals from the first two peaks in FTs (Fig. 2), corresponding mainly to the first two coordination shells around nickel, has been performed within the single-scattering approximation using conventional multi-component approach within the single-scattering approximation [14] as described in Section 2. Only the contributions from the first and second coordination shells, composed of oxygen $\left(\mathrm{O}_{1}\right)$ and nickel $\left(\mathrm{Ni}_{2}\right)$ atoms, respectively, were considered and resulted in good agreement between calculated and experimental EXAFS signals (Fig. 3). The obtained values of the structural parameters $\left(N, R, \sigma^{2}\right)$ are reported in Table 1. Note that the coordination numbers and interatomic distances in bulk c-NiO agree well with the known crystallographic values $\left(N_{\mathrm{XRD}}(\mathrm{O} 1)=6, R_{\mathrm{XRD}}\left(\mathrm{Ni}-\mathrm{O}_{1}\right)=2.089 \AA\right.$, and $N_{\mathrm{XRD}}\left(\mathrm{Ni}_{2}\right)=12$, $\left.R_{\mathrm{XRD}}\left(\mathrm{Ni}-\mathrm{Ni}_{2}\right)=2.954 \AA[16]\right)$.

In nano-NiO three effects can be observed: (i) the average coordination numbers $N$ decrease due to the under-coordinated atoms located at the nanocrystals surface; (ii) the interatomic distances behave differently in the first and the second shells the Ni- $\mathrm{O}_{1}$ distance decreases by $\sim 0.01 \AA$, whereas the $\mathrm{Ni}-\mathrm{Ni}_{2}$ increases by about the same amount; (iii) the MSRD values in both shells have larger values than in bulk c-NiO. Thus, one can conclude that the local structure in nano- $\mathrm{NiO}$ relaxes leading to an increase of disorder.

Detailed comparison of the total EXAFS signals in Fig. 2 allows one to notice that the frequency of oscillations is slightly higher in nano-NiO than in $\mathrm{c}-\mathrm{NiO}$. This fact reflect the difference in the average lattice parameters $\left(a_{0}\right)$ in two samples. Their values, estimated from $\mathrm{Ni}-\mathrm{Ni}_{2}$ distances, are equal to $a_{0}=4.188 \AA$ for nano-NiO and $a_{0}=4.171 \AA$ for c-NiO. The latter value agrees well with the x-ray diffraction results $a_{0}=4.1773 \AA$ for c-NiO [16]. Assuming the cubic shape for nanoparticles, one can evaluate the change in the lattice volume being $\Delta V / V_{0} \simeq 1 \%\left(V_{0}=72.5 \AA^{3}\right.$ for $\mathrm{c}-\mathrm{NiO}$ and $V=73.43 \AA^{3}$ for nano$\mathrm{NiO}$ ). Thus, our data confirm the expansion of the nano-NiO lattice, found recently by $\mathrm{x}$-ray diffraction $[10,11]$.

Further we will discuss the outer shells (beyond the second one) contributions into the total EXAFS signal. Their reliable analysis is a challenge, that requires correct account for the multiple-scattering (MS) effects. The peculiarity of the NiO crystal structure is responsible for the presence of many linear MS paths with large relative amplitude. Therefore, to address this problem, we used recently developed approach [12], allowing the calculation of the configuration-averaged EXAFS signal based on the results of the MD simulations.

First, we will discuss the results for bulk c-NiO. The MD simulations were performed using two FF potential models, developed in the past by Fisher [23] and Oliver [24]. The two models include the same pair interactions, but differ slightly in the numerical values of the FF parameters (Table 2).

In Fig. 5 the configuration-averaged EXAFS signals, calcu- 

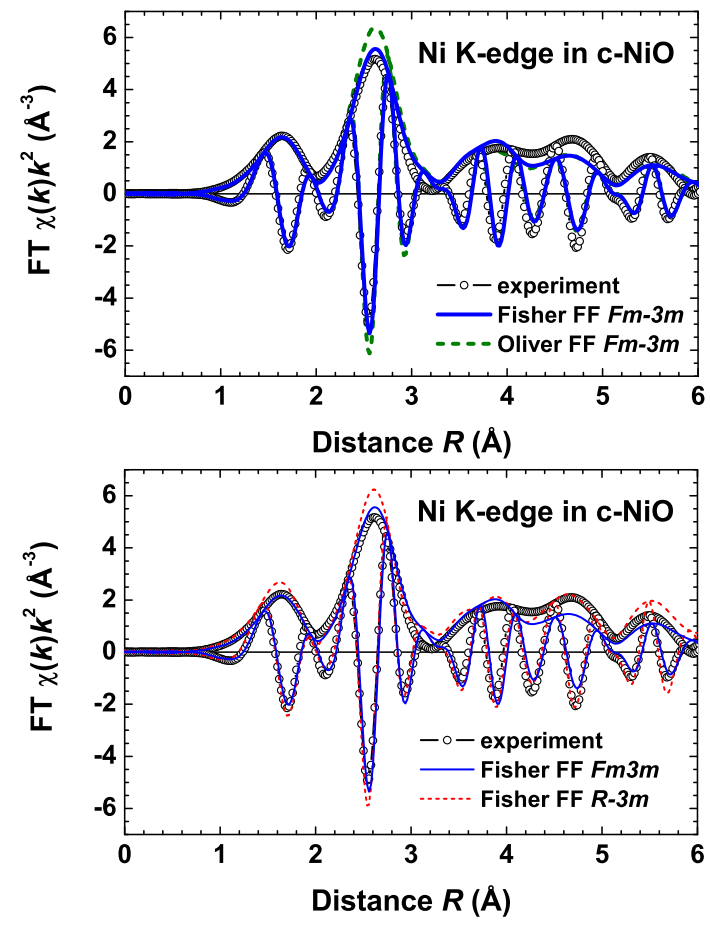

Figure 5: Comparison of experimental (open circles) and calculated Ni K-edge EXAFS $\chi(k) k^{2}$ signals and their Fourier transforms for c-NiO. Upper panel: simulations performed for the cubic $F m \overline{3} m \mathrm{c}-\mathrm{NiO}$ phase (solid line - Fisher's force field (FF) [23], dashed line - Oliver's FF [24]). Lower panel: simulations performed using Fisher's FF [23] for the cubic $F m \overline{3} m$ (solid line) and rhombohedral $R \overline{3} m$ (dashed line) c-NiO phases.

lated using two FF models, are compared with the experiment for c-NiO. The observed agreement is good, especially taking into account the simplicity of the models. Note that no fitting parameters were used in the calculations. More detailed comparison of the two models suggested that the Fisher's FF model [23] provides slightly better agreement in the FT region of the second peak at $\sim 2.6 \AA$; therefore only it will be considered further.

The simulations using the same Fisher's FF model were performed as for cubic $(F m \overline{3} m)$ as for rhombohedral $(R \overline{3} m) \mathrm{c}-\mathrm{NiO}$ phase (Fig. 5). Our results show that the rhombohedral distortion is presumably responsible for the high amplitude of the peak in FT at $4.7 \AA$ in the experimental signal.

The detailed contributions from single-scattering (SS) and multiple-scattering (MS) processes in $\mathrm{c}-\mathrm{NiO}$ and nano-NiO are shown in Figs. 6 and 7. As one can see, the MS signals are inevitably important in both materials in the region of the third peak at $3.8 \AA$ and of the fifth peak at $5.6 \AA$. Note that all signals in Figs. 6 and 7 entirely contain thermal disorder effects. Besides the EXAFS signal for nano-NiO, calculated for a particle size of $6 a_{0} \times 6 a_{0} \times 6 a_{0}$, takes into account both lattice expansion and surface termination effects. The agreement between calculated and experimental EXAFS signals in nano-NiO is good, however our simple FF model is not able to reproduce the relaxation of the first coordination shell in nano-NiO, found by the conventional analysis (Table 2).
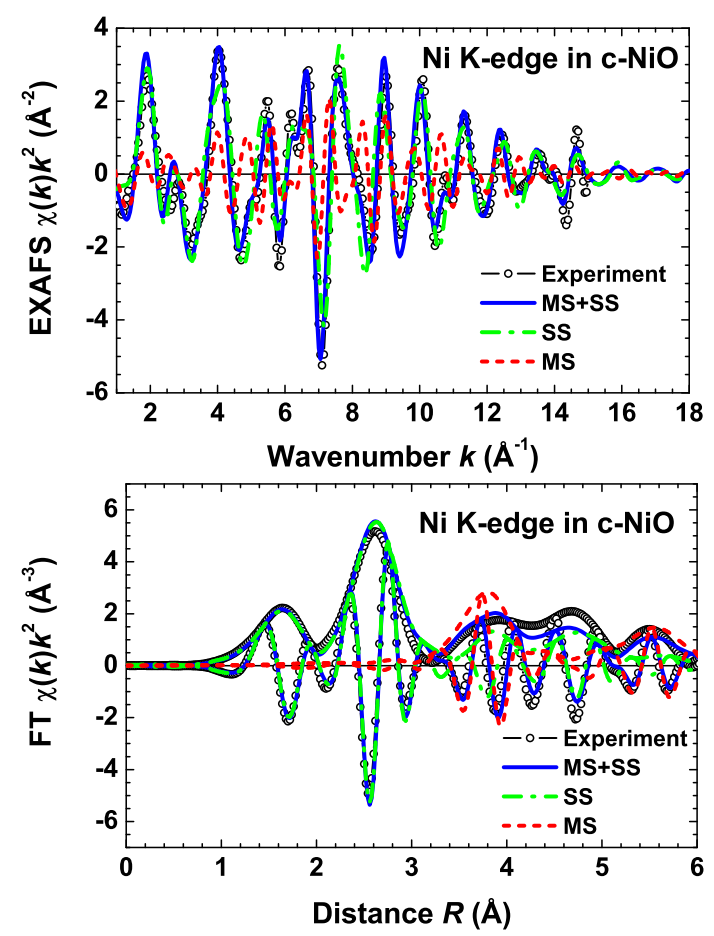

Figure 6: Comparison of experimental (open circles) and calculated (solid line) Ni K-edge EXAFS $\chi(k) k^{2}$ signals and their Fourier transforms for c-NiO. Dashed and dash-dot lines show multiple-scattering (MS) and single-scattering (SS) contributions into the total calculated EXAFS signal, respectively.
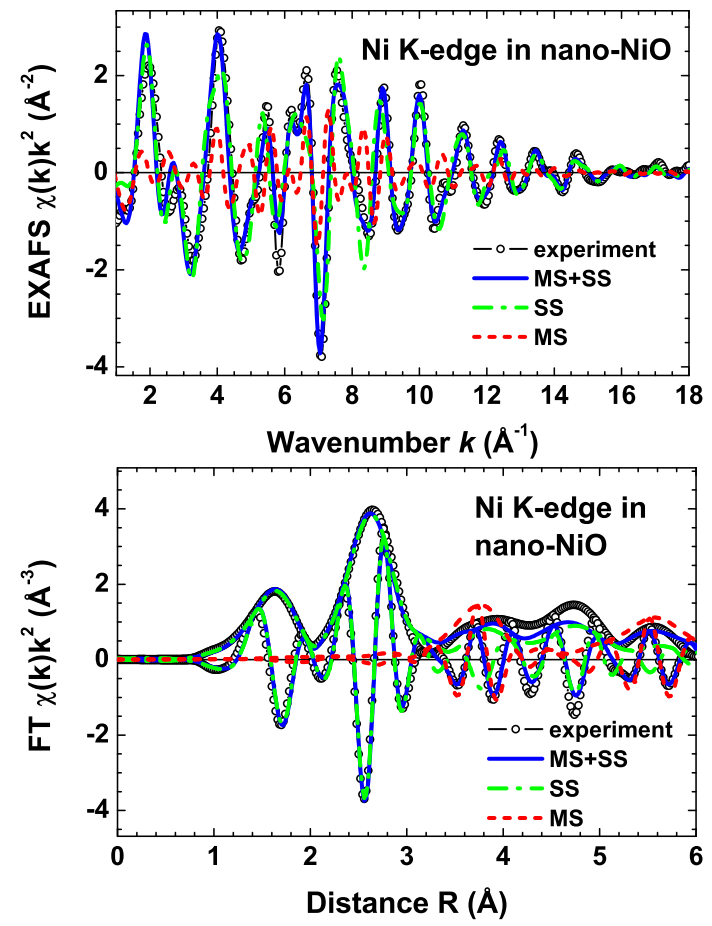

Figure 7: Comparison of experimental (open circles) and calculated (solid line) Ni K-edge EXAFS $\chi(k) k^{2}$ signals and their Fourier transforms for nano-NiO. Dashed and dash-dot lines show multiple-scattering (MS) and single-scattering (SS) contributions into the total calculated EXAFS signal, respectively. 
The RDFs $G(R)$ obtained during the MD runs for $\mathrm{c}-\mathrm{NiO}$ and nano-NiO (Fig. 4) were used to determine a set of structural parameters as coordination numbers, interatomic distances, and MSRDs for the first six coordination shells (Table 3). This was done by decomposition of $G(R)$ into a set of Gaussian functions. The obtained values for $\mathrm{c}-\mathrm{NiO}$ are in good agreement with crystallographic data. For nano-NiO, two sets of structural parameters were found, corresponding to the original [23] and optimized Fisher's force fields. The main difference between them is observed only in the values of interatomic distances, being due to different $\rho$ values in the Buckingham potential (Table 2). The coordination numbers $N$ in nano-NiO are reduced compared to c-NiO (Table 3): the effect increases with the interatomic distance due to the surface termination effect, as expected.

The significant difference is also observed between the MSRD values in nano-NiO and c-NiO. In the bulk, the MSRD describes the thermal disorder contribution. The thermal atoms motion is partially correlated for the nearest atoms, due to the direct chemical bonding, however, when the correlation effects in thermal motion at large distances become negligible, the MSRD value approaches towards a sum of the mean-squared displacements (MSD) for two atoms. Therefore, the MSRD for the atom pair $\mathrm{A}$ and $\mathrm{B}$ is given by

$$
\operatorname{MSRD}_{\mathrm{AB}}=\mathrm{MSD}_{\mathrm{A}}+\mathrm{MSD}_{\mathrm{B}}-\mathrm{DCF}_{\mathrm{AB}}
$$

where DCF is the displacement correlation function [35].

The MSD values can be experimentally determined from diffraction measurements: $\operatorname{MSD}(\mathrm{O})=0.0032 \AA^{2}$ and $\mathrm{MSD}(\mathrm{Ni})=0.0028 \AA^{2}$ for $\mathrm{c}-\mathrm{NiO}$ at $300 \mathrm{~K}[22]$. This means that the values of the MSRDs equal to $0.0060 \AA^{2}$ and $0.0056 \AA^{2}$ are expected for $\mathrm{Ni}-\mathrm{O}$ and $\mathrm{Ni}-\mathrm{Ni}$ atom pairs, respectively, for uncorrelated motion $(\mathrm{DCF}=0)$ at large distances. Note that these values are in good agreement with our simulation results for the fifth and sixth coordination shells (Table 3).

In nano-NiO, the softening of interaction potentials leads to the lattice expansion and to an increase of the amplitude of thermal vibrations (see optimized Fisher's FF model in (Table 3). Besides, the distribution of structural sites contributes additionally to an increase of the MSRDs since atoms located closer to the nanoparticle surface have vibration amplitude larger than the ones being closer to the nanoparticle center. Therefore, the average MSRDs are larger by about 0.002-0.004 $\AA^{2}$ in nano$\mathrm{NiO}$ compared to c-NiO (Table 3).

\section{Conclusions}

The local structure and it's relaxation around nickel atoms were studied in nano-sized nickel oxide (nano-NiO) by the $\mathrm{Ni}$ K-edge $\mathrm{x}$-ray absorption spectroscopy. We found that the lattice in nano-NiO expands compared to bulk nickel oxide (c-NiO), in agreement with other studies by EXAFS [28, 34] and XRD $[10,11]$. The estimate, based on the EXAFS signal analysis from the first two-coordination shells (Table 1), suggests a volume expansion in nano-NiO by about $1 \%$. At the same time, a contraction of the Ni-O bond by about $0.5 \%$ was observed in
Table 3: Structural parameters ( $N$ is the coordination number, $R$ is the interatomic distance, and $\sigma^{2}$ is the MSRD) for the first six coordination shells in $\mathrm{c}-\mathrm{NiO}$ and nano-NiO obtained by decomposition of the Ni-O and Ni-Ni RDFs into Gaussian components.

\begin{tabular}{|c|c|c|c|c|c|c|}
\hline & $\mathrm{O}_{1}$ & $\mathrm{Ni}_{2}$ & $\mathrm{O}_{3}$ & $\mathrm{Ni}_{4}$ & $\mathrm{O}_{5}$ & $\mathrm{Ni}_{6}$ \\
\hline & \multicolumn{6}{|c|}{$\mathrm{c}-\mathrm{NiO}$} \\
\hline$N$ & 6 & 12 & 8 & 6 & 24 & 24 \\
\hline$R(\AA)$ & 2.083 & 2.949 & 3.610 & 4.167 & 4.661 & 5.106 \\
\hline \multirow[t]{2}{*}{$\sigma^{2}\left(\AA^{2}\right)$} & 0.0055 & 0.0042 & 0.0059 & 0.0056 & 0.0062 & 0.0057 \\
\hline & \multicolumn{6}{|c|}{ nano-NiO (optimized Fisher’s FF) } \\
\hline$N$ & 5.4 & 10.0 & 6.1 & 5.0 & 18.3 & 16.8 \\
\hline$R(\AA)$ & 2.092 & 2.970 & 3.647 & 4.185 & 4.688 & 5.147 \\
\hline \multirow[t]{2}{*}{$\sigma^{2}\left(\AA^{2}\right)$} & 0.0071 & 0.0063 & 0.0075 & 0.0104 & 0.0101 & 0.0097 \\
\hline & \multicolumn{6}{|c|}{ nano-NiO (Fisher’s FF [23]) } \\
\hline$N$ & 5.4 & 10.0 & 6.1 & 5.0 & 18.3 & 16.8 \\
\hline$R(\AA)$ & 2.066 & 2.933 & 3.601 & 4.132 & 4.629 & 5.082 \\
\hline$\sigma^{2}\left(\AA^{2}\right)$ & 0.0069 & 0.0060 & 0.0072 & 0.0101 & 0.0099 & 0.0093 \\
\hline$N_{\text {nano }} / N_{\text {cryst }}$ & 0.90 & 0.83 & 0.76 & 0.83 & 0.76 & 0.70 \\
\hline
\end{tabular}

the first coordination shell. Note that similar effect has been previously detected in $\mathrm{NiO}$ thin films [28, 34].

The full EXAFS signals in both c-NiO and nano-NiO were analysed by a recently developed approach [12], based on a combination of classical molecular dynamics (MD) and ab initio multiple-scattering EXAFS theory. The two force-field (FF) models by Fisher [23] and Oliver [24] were applied to c-NiO (Fig. 5 and Table 2). The results obtained with the Fisher's FF model provided better agreement between configurationaveraged and experimental Ni K-edge EXAFS signals; therefore it was used further for the simulation of nano-NiO.

While most simulations were performed using cubic NiO lattice as a starting point, we have also checked the influence of the magnetostriction effect on the EXAFS signal (Fig. 5). Our results show that the rhombohedral distortion, occurring in bulk c-NiO at $300 \mathrm{~K}$, is presumably responsible for the high amplitude of the peak in the Fourier transforms of the EXAFS signals at $4.7 \AA$.

In nanosized $\mathrm{NiO}$, we found that original Fisher's FF model fails to reproduce the lattice expansion and total EXAFS signal (Fig. 6). However, the model can be corrected by adjusting the $\rho$ parameter of the Buckingham potentials (Table 2) to make them more repulsive.

To conclude, we showed that the MD-EXAFS simulations of the $\mathrm{Ni} \mathrm{K}$-edge in $\mathrm{c}-\mathrm{NiO}$ and nano-NiO, based on a simple rigid-ion force-field model, provide good agreement with the experiment. Such approach requires less parameters than conventional EXAFS analysis and allows accounting explicitly for thermal effects and many-atom distribution functions. The agreement between the experimental and calculated EXAFS signals can be used as a criterion to optimize the parameters of the force-field model. 


\section{Acknowledgements}

This work was supported by ESF Project 2009/0202/1DP/1.1.1.2.0/09/APIA/VIAA/141 and Latvian Government Research Grant No. 09.1518. The research leading to these results has received funding from the European Community's Seventh Framework Programme (FP7/20072013) under grant agreement No. 226716 (Projects I-20090071 EC and I-20100110 EC).

\section{References}

[1] S. J. L. Billinge, I. Levin, Science 316 (2007) 561.

[2] S. J. L. Billinge, J. Solid State Chem. 181 (2008) 1695.

[3] J. J. Rehr, R. C. Albers, Rev. Mod. Phys. 72 (2000) 621.

[4] C. Q. Sun, Prog. Solid State Chem. 35 (2007) 1.

[5] S. Tsunekawa, K. Ishikawa, Z.-Q. Li, Y. Kawazoe, A. Kasuya, Phys. Rev. Lett. 85 (2000) 3440.

[6] M. Fukuhara, Phys. Lett. A 313 (2003) 427.

[7] G. Li, J. Boerio-Goates, B.F. Woodfield, L. Li, Appl. Phys. Lett. 85 (2004) 2059.

[8] A. Kossoy, Y. Feldman, E. Wachtel, K. Gartsman, I. Lubomirsky, J. Fleig, J. Maier, Phys. Chem. Chem. Phys. 8 (2006) 1111.

[9] R. N. Bhowmik, R. Ranganathan, R. Nagarajan, Phys. Rev. B 73 (2006) 144413.

[10] L. Li, L. Chen, R. Qihe, G. Li, Appl. Phys. Lett. 89 (2006) 134102.

[11] M. Ghosh, K. Biswas, A. Sundaresana, C. N. R. Rao, J. Mater. Chem. 16 (2006) 106

[12] A.Kuzmin, R.A. Evarestov, J. Phys.: Condens. Matter 21 (2009) 055401.

[13] A. Kuzmin, Physica B 208/209 (1995) 175.

[14] V. L. Aksenov, M. V. Kovalchuk, A. Yu. Kuzmin, Yu. Purans, S. I. Tyutyunnikov, Cryst. Rep. 51 (2006) 908.

[15] A. L. Ankudinov, B. Ravel, J. J. Rehr, S. D. Conradson, Phys. Rev. B 58 (1998) 7565 .

[16] A. Kuzmin, N. Mironova, J. Phys.: Condens. Matter 10 (1998) 7937.

[17] A. Kuzmin, R. A. Evarestov, J. Phys.: Conf. Series 190 (2009) 012024.

[18] S. Brunauer, P. H. Emmett, E. Teller, J. Am. Chem. Soc. 60 (1938) 309.

[19] A. Kalinko, R. A. Evarestov, A. Kuzmin, J. Purans, J. Phys.: Conf. Series 190 (2009) 012080.

[20] J. D. Gale, A. L. Rohl, Mol. Simul. 9 (2003) 291.

[21] W. L. Roth, Phys. Rev. 110 (1958) 1333.

[22] D. Rodic, V. Spasojevic, V. Kusigerski, R. Tellgren, H. Rundlof, Phys. Status Solidi (b) 218 (2000) 527.

[23] C. A. J. Fisher, Scripta Materiala 50 (2004) 1045.

[24] P. M. Oliver, G. W. Watson, S. C. Parker, Phys. Rev. B 52 (1995) 5323.

[25] A. Kuzmin, Latv. J. Phys. Tech. Sci. 2 (2006) 7.

[26] P. Kizler, Phys. Rev. B 46 (1992) 10540.

[27] J. P. Hill, C.-C. Kao, D. F. McMorrow, Phys. Rev. B 55 (1997) R8662.

[28] A. Kuzmin, J. Purans, A. Rodionov, J. Phys.: Condens. Matter 9 (1997) 6979.

[29] R. V. Vedrinskii, V. L. Kraizman, A. A. Novakovich, Sh. M. Elyafi, S Bocharov, Th. Kirchner, G. Dräger, Phys. Status Solidi (b) 226 (2001) 203.

[30] H. Modrow, S. Bucher, J. J. Rehr, A. L. Ankudinov, Phys. Rev. B 67 (2003) 035123.

[31] Z. Y. Wu, D. C. Xian, T. D. Hu, Y. N. Xie, Y. Tao, C. R. Natoli, E. Paris, A. Marcelli, Phys. Rev. B 70 (2004) 033104.

[32] A. Kuzmin, J. Purans, R. Kalendarev, Phys. Status Solidi (c) 2 (2005) 665.

[33] C. Gougoussis, M. Calandra, A. Seitsonen, Ch. Brouder, A. Shukla, F. Mauri, Phys. Rev. B 79 (2009) 045118.

[34] E. Avendano, A. Kuzmin, J. Purans, A. Azens, G. A. Niklasson, C.G. Granqvist, Physica Scripta T115 (2005) 464.

[35] G. Beni, P. M. Platzman, Phys. Rev. B 14 (1976) 1514. 